Con questo numero del GTN\&D inauguriamo una rubrica su argomenti riguardanti la dialisi peritoneale. Ci sembra possa essere utile perché si parla e si scrive poco di dialisi peritoneale e perché ci proponiamo di raggiungere e interessare i molti operatori che continuano a considerarla un trattamento per pochi pazienti del quale è sufficiente che si occupi qualcun altro. Naturalmente nella posizione di questi operatori c'è un fondamento di verità perché i programmi di dialisi peritoneale coinvolgono meno operatori sanitari rispetto all'emodialisi e perché i pazienti per lo più stanno a casa e frequentano poco l'ospedale. Questi, che per noi sono pregi della tecnica, in realtà ne costituiscono anche il tallone di Achille, perché la scarsa diffusione della peritoneale e delle relative competenze tra gli operatori contribuiscono a rendere più "fragile" sia la metodica che lo stesso paziente.

Su ogni numero del GTN\&D faremo brevemente il punto su un argomento prendendo spunto dalla letteratura recente. Inoltre, ci saranno spazi dedicati ad eventuali novità normative e all'attività del Gruppo di Studio di Dialisi Peritoneale della Società Italiana di Nefrologia.

\title{
Quando è meglio rimuovere il catetere peritoneale dopo trapianto renale?
}

\author{
Gianna d'Adamo
}

\author{
Nefrologa, Roma
}

\begin{abstract}
TO LEAVE OR TO REMOVE PERITONEAL CATHETERS AT THE TIME OF RENAL TRANSPLANTATION?
Abstracts. There is no consensus with regard to the timing of PD catheter removal in patients undergoing renal transplantation. The European Best Practice Guidelines recommended leaving the PD catheter in place in the event of delayed graft function. However, the high rates of PD-related failure/complications in the post-transplant setting induced some Centers to remove the PD catheter at the time of transplant surgery as to avoid potential complications and the need for a second procedure.
\end{abstract}

Key words: Peritoneal dialysis catheter, Kidney transplant

Conflict of interest: None.

Ricevuto: 11 Gennaio, 2013; Accettato: 26 Gennaio 2013

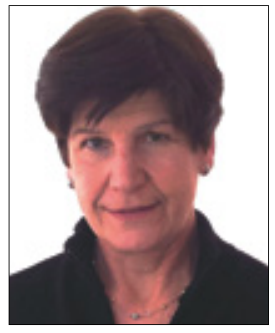

Gianna d'Adamo
A proposito della dialisi peritoneale (DP) dopo trapianto, le linee guida europee (1) formulano le seguenti 3 raccomandazioni, tutte derivanti da studi clinici ben condotti, ma non da trials controllati randomizzati (livello B di "evidence"):

1. La DP può essere usata in corso di ritardata ripresa funzionale (DGF) del nuovo rene;

2. Il catetere può essere lasciato in sede per 3-4 mesi con trapianto funzionante; è tuttavia consigliabile rimuoverlo prima, quando il decorso post trapianto è favorevole;

3. Peritoniti ed infezioni dell'exit site andrebbero trattate secondo le linee guida ISPD, abbassando però la soglia per la rimozione del catetere.

Nel razionale delle linee guida si specifica che l'incidenza e la gravità di DGF sono minori nei riceventi che provengono dalla DP rispetto a quelli provenienti dall'emodialisi e che, in caso di necessità dialitica post-trapianto, è comunque possibile utilizzare la DP se la cavità peritoneale non è stata aperta durante l'intervento. La maggiore incidenza di peritoniti e infezioni dell'exit site, rispetto al periodo pre-trapianto, sarebbe controbilanciata dal rischio di batteriemia da catetere per emodialisi, qualora si renda necessario un supporto dialitico in corso di terapia immunosoppressiva. L'esperienza di due Centri Trapianto recentemente pubblicata da J. Warren sul Canadian Urological Association Journal (CUAJ) propone alcune ulteriori riflessioni sull'argomento (2). La casistica comprende 137 pazienti in DP sottoposti a trapianto di rene nel periodo 2005-2008. 
In 19 casi il catetere peritoneale era stato rimosso al momento del trapianto e non si erano osservate complicanze correlate con la rimozione né con l'emodialisi resasi necessaria in un caso per DGF. Negli altri 118 pazienti con catetere peritoneale in situ si sono invece osservate complicanze, anche senza l'utilizzo del catetere. Infatti, su 103 pazienti $(89 \%)$ con pronta ripresa funzionale del rene trapiantato, si erano avute 2 infezioni dell'exit site e 5 peritoniti. Dei 15 pazienti $(12,7 \%)$ con necessità dialitica per DGF, ben 3 non avevano potuto effettuare DP per stravaso di soluzione dialitica dalla ferita chirurgica e 5 avevano sviluppato peritonite.

A seguito di queste osservazioni e per evitare la necessità di una successiva ulteriore anestesia, gli autori propongono di rimuovere il catetere contestualmente al trapianto e sottolineano l'opportunità di studi volti ad identificare i casi che possono trarre effettivo beneficio dalla permanenza in situ del catetere peritoneale, in base alle caratteristiche del ricevente e alle condizioni del donatore.

Sullo stesso numero del CUAJ, Tom McGregor segnala che l'esperienza del Centro di Winnipeg è stata molto simile a quella pubblicata da Warren, riassume pro (possibilità di uso del catetere in caso di DGF ) e contro (potenziali complicanze del catetere, necessità di una seconda procedura) del mantenimento del catetere peritoneale al momento del trapianto di rene e considera minimi i benefici, soprattutto in caso di donatore vivente (3).

Il dibattito sull'argomento è dunque ancora aperto ma l'alto tasso di complicanze segnalato dai lavori citati sembra comunque indicare l'opportunità che la cura del catetere e l'eventuale DP post-trapianto siano affidati a personale con competenza ed esperienza specifiche.

\section{Riassunto}

Il dibattito sull'opportunità o meno di rimuovere il catetere peritoneale all'atto del trapianto renale è ancora aperto. Il mantenimento in sede del catetere viene infatti raccomandato dalle linee guida europee del 2005 per consentire la ripresa della dialisi peritoneale in caso di ritardata ripresa funzionale del rene trapiantato. Alcuni Centri Trapianto hanno però recentemente segnalato un alto tasso di complicanze post-trapianto connesse con la presenza del catetere, anche se non utilizzato, e suggeriscono di rimuoverlo al momento del trapianto per abbattere il rischio di complicanze e/o fallimento della DP ed evitare una seconda procedura a breve distanza dal trapianto.

Parole chiave: Catetere peritoneale, Trapianto renale

Dichiarazione di conflitto di interessi: L'Autore dichiara di non avere conflitto di interessi.

Indirizzo degli Autori:

Dr.ssa Gianna d'Adamo

Nefrologa

Roma

giannadadamo@gmail.com

\section{Bibliografia}

1. Dombros N, Dratwa M, Feriani M, et al. European best practice guidelines for peritoneal dialysis. 3 Peritoneal access. Nephrol Dial Transplant 2005; 20: ix8-ix12.
2. Warren J, Jones E, Sener A, et al. Should peritoneal dialysis catheters be removed at the time of kidney transplantation? Can Urol Assoc J 2012; 6: 376-8.

3. McGregor T. The PD catheter dilemma. Can Urol Assoc J 2012; 6: 379 . 